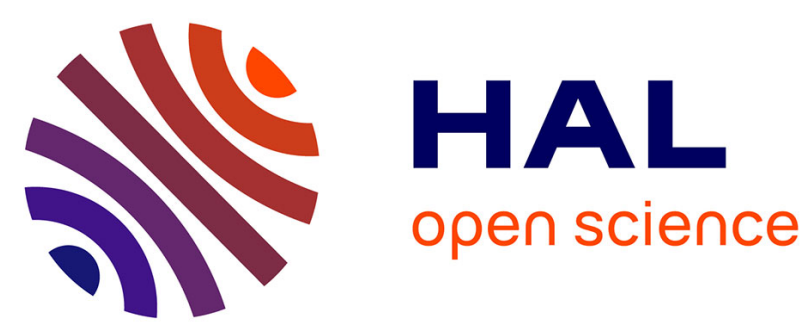

\title{
Radiobiologic Parameters and Local Effect Model Predictions for Head-and-Neck Squamous Cell Carcinomas Exposed to High Linear Energy Transfer Ions
}

M. Beuve, G. Alphonse, M. Maalouf, A. Colliaux, P. Battiston-Montagne, P. Jalade, E. Balanzat, A. Demeyer, M. Bajard, C. Rodriguez-Lafrasse

\section{To cite this version:}

M. Beuve, G. Alphonse, M. Maalouf, A. Colliaux, P. Battiston-Montagne, et al.. Radiobiologic Parameters and Local Effect Model Predictions for Head-and-Neck Squamous Cell Carcinomas Exposed to High Linear Energy Transfer Ions. International Journal of Radiation Oncology, Biology, Physics, 2008, 71, pp.635-642. 10.1016/j.ijrobp.2007.10.050 . in2p3-00321614

\section{HAL Id: in2p3-00321614 https://hal.in2p3.fr/in2p3-00321614}

Submitted on 16 Sep 2008

HAL is a multi-disciplinary open access archive for the deposit and dissemination of scientific research documents, whether they are published or not. The documents may come from teaching and research institutions in France or abroad, or from public or private research centers.
L'archive ouverte pluridisciplinaire HAL, est destinée au dépôt et à la diffusion de documents scientifiques de niveau recherche, publiés ou non, émanant des établissements d'enseignement et de recherche français ou étrangers, des laboratoires publics ou privés. 


\title{
RADIOBIOLOGICAL PARAMETERS AND LOCAL EFFECT MODEL PREDICTIONS FOR HEAD AND NECK SQUAMOUS CARCINOMA CELLS EXPOSED TO HIGH LINEAR ENERGY TRANSFER IONS
}

\author{
Michael Beuve, PhD ${ }^{1}$, Gersende Alphonse, PhD ${ }^{2,3}$, Mira Mahlouf ${ }^{2}$, Anthony \\ Colliaux ${ }^{1}$, Priscilla Battiston-Montagne ${ }^{2}$, Patrice Jalade, PhD ${ }^{1,3}$, Emmanuel \\ Balanzat $^{4}$, Albert Demeyer, PhD ${ }^{1}$, Marcel Bajard, PhD ${ }^{1}$, Claire Rodriguez- \\ LAFrasse, PHD $^{2}$
}

${ }^{1}$ IPNL, IN2P3, Université Lyon 1, Villeurbanne, F-69622, France; ${ }^{2}$ Laboratoire de Radiobiologie Cellulaire et Moléculaire, EA 3738, Faculté de Médecine Lyon-Sud, Université Lyon 1, Oullins, F-69921, France; Université de Lyon, Lyon, F-69003, France; ${ }^{3}$ Hospices Civils de Lyon, Service de Radiothérapie, Centre Hospitalier Lyon-Sud, Pierre Bénite, F69495, France; ${ }^{4}$ CIRIL, Caen, F-14070, France.

Request for reprints: Dr Michael BEUVE, IPNL, Bâtiment DIRAC, 43, bd du 11 novembre 1918, 69622 Villeurbanne cedex, France. Phone: 003347244 79 36; fax 00334724315 36; e-mail: mbeuve@ipnl.in2p3.fr

Running title: Radiobiology and LEM predictions to high LET ions for HNSCC

\section{Acknowledgments:}

We thank all who participated in experiments at GANIL or GSI: Marie-Thérèse Aloy, Christophe Badie, Clara Bionda, Anthony Boivin, Jacques Bodennec, Djamel Dabli, Francis Djoumessi, Elie Hadchity, Nelly Loupeda, Marie-Charlotte Ricol and Selena TrajkovicBodennec. We also thank Joel Rochat, Joseph Remillieux and Jacques Balosso for their continued assistance in accomplishing this work. We are grateful to the personnel of the 
radiotherapy department (under Dr P. Romestaing) for allowing us access to the gamma irradiators of the Lyon-Sud Hospital Center. We also wish to thank the staff of the CIRIL (GANIL, Caen, France) and Biophysics (GSI, Darmstadt, Germany) laboratories for their help in experiments.

This work was achieved within the scientific framework of the French ETOILE project of hadrontherapy and supported in part by the Région Rhône-Alpes and the Communauté Urbaine de Lyon. Anthony Colliaux was funded by the Institut National du Cancer (INCA). 


\begin{abstract}
Purpose: To establish the radiobiological parameters of head-and-neck squamous carcinoma cells (HNSCC) in response to ion irradiation with various linear energy transfer (LET) values and to evaluate the relevance of the local effect model (LEM) in HNSCC.

Methods: Cell survival curves were established in radiosensitive SCC61 and radioresistant

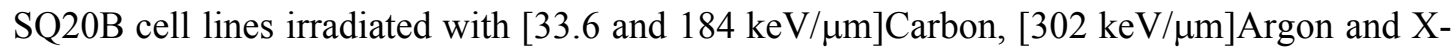
rays. The results of ion experiments were confronted to LEM predictions.

Results: The relative biological efficiency ranged from 1.4 to 4.0 for SCC61 and 2.1 to 3.0 for SQ20B cells. Fixing an arbitrary $\mathrm{D}_{0}$ parameter, which characterized survival to X-ray at high doses ( $>10 \mathrm{~Gy}$ ), gave unsatisfying LEM predictions for both cell lines. For $\mathrm{D}_{0}=10 \mathrm{~Gy}$, the error on survival fraction at $2 \mathrm{~Gy}$ amounted to a factor of 10 for $[184 \mathrm{keV} / \mu \mathrm{m}]$ Carbon in SCC61 cells. We showed that the slope $\left(s_{\max }\right)$ of the survival curve at high doses was much more reliable than $\mathrm{D}_{0}$. Fitting $s_{\max }$ to $2.5 \mathrm{~Gy}^{-1}$ gave better predictions for both cell lines. Nevertheless, LEM could not predict the responses to fast and slow ions with the same accuracy.

Conclusions: The LEM could predict the main trends of these experimental data with correct orders of magnitude while $s_{\max }$ was optimized. Thus, the efficiency of carbon ions cannot be simply extracted from the clinical response of a patient to X-rays. LEM should help to optimize planning for hadrontherapy if a set of experimental data is available for high-LET radiations in various types of tumors.
\end{abstract}

Key Words: High-LET Ion, Carbon Irradiation, Argon Irradiation, Local Effect Model, Head-and-Neck Squamous Carcinoma Cells.

\title{
No conflict of interest.
}




\section{INTRODUCTION}

Hadrontherapy by carbon ions is clearly efficient to cure tumors resistant to conventional radiotherapy (1-3) because of the specific physical and biological properties of light ions. Very precise lateral and longitudinal dose profiles can be achieved even at large penetration depths (4) and doses delivered to the patient can be controlled by positron emission tomography (5). The biological efficiency stems from a low effect at the entrance in healthy tissues and a high relative biological effect (RBE) at the tumor position (4). Therefore, treatment planning has to integrate physical parameters, biological parameters and models to optimize tumor control. Hence, the local effect model (LEM) aims at extrapolating to hadrontherapy all the knowledge accumulated in the case of conventional radiotherapy. Comparisons with experimental data demonstrate that LEM is well suited to predict survival in various healthy mammalian cell lines (6). However, to date it seems that LEM has not been studied extensively in human tumor cells (7).

The aim of this study was to evaluate the prediction of LEM in two human squamous carcinoma cell lines derived from tumor of the pharynx (SCC61) and neck (SQ20B) that display opposite sensitivity to photon irradiation. Cell survival was chosen as the endpoint among others, considering its correlation with tumor regression. Standardization of experimental protocols for both photon and ion irradiation (argon and carbon) allowed us to establish conclusions concerning our evaluation of LEM.

\section{MATERIALS AND METHODS}

\section{Local effect model}

The LEM model stands in the framework of "track structure" models (8), which considers that the particular features of ion-induced biological effects, such as relative biological effect (RBE) or the presence of hooks in inactivation cross-sections (9), stems from the strong heterogeneity of the deposited energy. While some models (10) clearly separate the irradiated volume into intratrack and intertrack components, respectively responsible for "ion-kill" and "gamma-kill" events, the LEM proposes instead a formulation simply based on the local energy distribution and the response to X-rays. The main points of LEM, summarized below, are described in (11).

By definition, the survival probability is the probability that a cell does not undergo any lethal event. Assuming a Poisson distribution for lethal event numbers, the mean number of induced lethal events is: 


$$
N_{\text {lethal }}(D)=-\ln [S(D)]
$$

where $S(D)$ is the surviving probability at dose $D$.

The LEM assumes that $N_{\text {lethal }}$ is in fact a local quantity generated by the local dose. For Xray radiation, which is assumed homogenous throughout the cell, the lethal event probability is homogenous in the sensitive volume $V_{n}$ of the cell. The sensitive volume is restricted to the cell nucleus described by its radius. For ion irradiation, the local dose is highly heterogeneous. The mean number of lethal events results from the contribution of each local event:

$$
N_{\text {lethal }}=\iiint_{J_{N}}-\frac{\ln \left[S_{X}(D(r))\right]}{V_{N}} d r
$$

The local dose $D(r)$ is the superimposition of the dose deposited by each impinging ion for a given irradiation configuration. Because of the radial symmetry of track structure, the dose deposited by each ion is approximated by a radial dose:

$$
\left\{\begin{array}{l}
D(r)=\frac{\lambda}{r_{\min }^{2}} \text { for } r<r_{\min } \\
D(r)=\frac{\lambda}{r^{2}} \text { for } \quad r_{\min } \leq r<r_{\max }
\end{array}\right.
$$

where $r$ is the distance to the ion trajectory.

The radial dose is an important parameter because it contains the ion's atomic number and energy. In particular, it includes the LET value through the normalization coefficient $\lambda$. We used in our case LET values provided by the code SRIM 2003 (12). While $r_{\min }$ is fixed to 10 $\mathrm{nm}, r_{\max }$, the maximal distance ranged by the delta electrons, depends on the ion energy. The survival probability is derived from Eq. (1). To be compared with the experimental value at ion dose $D_{i o n}$, the calculated survival is averaged by Monte Carlo simulation over many irradiation configurations. Although local doses can reach very high levels, survival curves can only be measured for doses of X-ray lower than 10 Gy. Therefore, the linear quadratic (LQ) model, which is used to describe cell survival to X-rays, is extrapolated continuously and linearly beyond a threshold dose, $\mathrm{D}_{0}$ and both the function and its derivative are continuous. 


$$
\left\{\begin{aligned}
-\ln \left[S_{X}(D)\right] & =\alpha D+\beta D^{2} \quad \text { for } \quad D<D_{0} \\
& =s_{\max }\left(D-D_{0}\right)-\ln \left[S\left(D_{0}\right)\right] \text { for } D>D_{0} \text { with } s_{\max }=\alpha+2 . \beta \cdot D_{0}
\end{aligned}\right.
$$

\section{Cell culture and nuclear size measurement}

The SCC61 and SQ20B cell lines were established respectively from squamous cell carcinomas of the pharynx and neck as described (13) and cultured as previously reported (14). To measure the sizes of nuclei, cells were grown on coverslips for $48 \mathrm{~h}$, fixed with $2.5 \%$ paraformaldehyde, permeabilized with Triton X 100 and stained with SYTOX Green nucleic acid stain (Invitrogen, Carlsbad, CA) before visualization using confocal microscopy (Centre technologique des microstructures, Université Lyon1, France). The mean nuclear surface area (S) was measured for 1000 cells of each cell line using standard image analysis software and the radius (R) was determined according to $R=\sqrt{S / \pi}$.

\section{Irradiation procedures}

Monolayers of cultured cells were irradiated with carbon or argon particles at fixed LET (track-segment protocol) and fixed ion energy. We irradiated at GANIL (Grand Accelérateur National d'Ions Lourds, Caen, France) with C[75 MeV/n] and $\operatorname{Ar}[95 \mathrm{MeV} / \mathrm{n}]$ and at GSI (Gesellschaft für SchwerIonenforschung, Darmstadt, Germany) with C[11.4 MeV/n]. Taking into account the traversal by the projectiles of the vacuum windows $(20 \mu \mathrm{m}$ Kapton at GSI and $10 \mu \mathrm{m}$ stainless in a G4 room at GANIL) and the flasks' front face $\left(0.124 \mathrm{~g} / \mathrm{cm}^{2}\right)$ for GANIL experiments, the LET and ion energy (SRIM 2003 code calculation (12)) in the cells were $33.6 \mathrm{keV} / \mu \mathrm{m} \mathrm{C}[72 \mathrm{MeV} / \mathrm{n}], 184 \mathrm{keV} / \mu \mathrm{m} \mathrm{C}[9.8 \mathrm{MeV} / \mathrm{n}]$ and $302 \mathrm{keV} / \mu \mathrm{m} \operatorname{Ar}[85$ $\mathrm{MeV} / \mathrm{n}]$, respectively.

Although experiments with high-velocity ions (experiments at GANIL) can be achieved in standard culture flasks (Method 1), the lowest LET carbon study required a specific experimental setup and protocol (Method 2). The ranges in water of $\operatorname{Ar}[85 \mathrm{MeV} / \mathrm{n}]$ and $C[72$ $\mathrm{MeV} / \mathrm{n}]$ beams are $6 \mathrm{~mm}$ and $17 \mathrm{~mm}$, respectively, compared with the $0.4 \mathrm{~mm}$ range of C[9.8 $\mathrm{MeV} / \mathrm{n}]$. X-ray survival curves were therefore established for both protocols.

\section{Photon irradiation :}

Method 1: Ten to sixteen hours before irradiation, SCC61 and SQ20B cells were seeded in ten flasks of $12.5 \mathrm{~cm}^{2}$ at different densities, depending on the dose of radiation. Cells were irradiated at room temperature on a Clinac $\mathrm{CD}$ irradiator with a photon energy of $10 \mathrm{MV}$, at doses of $0.5,1,1.5,2,2.5,3,4,5$ or $6 \mathrm{~Gy}$, delivered at a dose rate of $2 \mathrm{~Gy} / \mathrm{min}$. The 
irradiations were performed under clinical conditions in the radiotherapy department of LyonSud hospital. The ionization chambers used for dose measurements were calibrated according to international protocols.

Method 2: Cells were plated 10 to 16 hours before irradiation. Unlike Method 1, both cell lines were seeded at one unique concentration in Petri dishes $\left(36.3 \mathrm{~cm}^{2}\right.$ area). Irradiations were performed at GSI with $250 \mathrm{kV}$ X-rays at a dose rate of $1 \mathrm{~Gy} / \mathrm{min}$ with doses ranging from 0.5 to $6 \mathrm{~Gy}$.

C [72 MeV/n] and $\operatorname{Ar}[85 \mathrm{MeV} / \mathrm{n}]$ : For both ions, irradiations were performed in the G4 room of GANIL for which the irradiation field can reach up to $4 \times 40 \mathrm{~cm}$. The seeding procedure was the same as described in method 1. The inoculated SQ20B and SCC61 cells number was determined with respect to the platting efficiency and the expected survival, to obtain 50 to 100 colonies. In G4 room, four $12.5 \mathrm{~cm}^{2}$ flasks were irradiated vertically at the same time with varying between 0.5 to $6 \mathrm{~Gy}$ at a constant dose rate of $2 \mathrm{~Gy} / \mathrm{min}$. Two calibrated ionization chambers hanging close to the samples, were used to monitor the absolute dose. At low dose, we compared the dose measurement to fluence measurement using a nuclear track detector (CR39) (15). As expected, the ratio dose over fluence gave a stopping power value to within $6 \%$, in agreement with the tables published by Ziegler (12). To ensure a uniform constant dose, X-Omat films were intercalated systematically between the beam and samples. Any colony that developed outside the beam area for which the dose error was higher than $10 \%$ was disregarded.

$\mathbf{C}[9.8 \mathrm{MeV} / \mathbf{n}]$ : Irradiations were performed at GSI with the UNILAC linear accelerator. The protocol for irradiation and dose measurement was described in (16). As in method 2 aliquots of $3 \times 10^{5}$ SCC61 or SQ20B cells were seeded in Petri dishes and irradiated at doses varying between 0 and 6 Gy. Petri dishes containing cell cultures were opened and placed in a magazine full of sterile medium without fetal calf serum (FCS). During irradiation, a robot took the dishes one by one and placed the cells directly in front of the beam. At this energy, the ion range is quite low and particular care is required to ensure there is no significant beam straggling. The exit vacuum window consisted of a Kapton foil. The Petri dishes were opened and just covered by a thin film of culture medium. After irradiation, cells were trypsinized and reseeded with medium containing 10\% FCS at appropriate concentration in six $25 \mathrm{~cm}^{2}$ flasks before they were returned to the incubator. Beam homogeneity was controlled before experiments through the observation of a $\mathrm{ZnS}$ screen directly set in the beam. Absolute dose was monitored using an ionization chamber. A comparison of measured dose with CR39 
nuclear track detectors exhibited a linear dose response for a fluence range of $10^{3}$ to $10^{7}$ particles $/ \mathrm{cm}^{2}$.

\section{Cell survival assay}

Cell survival was assessed by a standard colony formation assay (17). Ten days after irradiation, cells were washed twice with PBS, fixed with ethanol and stained with a 5\% Giemsa solution. Colonies containing more than $2^{6}$ cells were scored to calculate the surviving fractions $\mathrm{S}(\mathrm{D})$ at each dose $(\mathrm{D})$ according to:

$$
S(D)=\frac{n(D)}{P E \cdot N(D)}
$$

where $n$ represents the colony number, $N$ the seeded-cell number and $P E$ the plating efficiency.

\section{Statistical analysis}

The accuracy of LEM predictions depends on the $\alpha$ and $\beta$ parameters of the LQ model. However, we observed that these values were very sensitive to fluctuations in experimental data. We therefore undertook a statistical analysis, developed an optimized estimator $P$ for survival probability and obtained:

$$
P=\sum_{i=1}^{q} \alpha_{i} \frac{n_{i}}{N_{i}}
$$

with:

$$
\alpha_{i}=\sum_{i=1}^{q} n_{i}
$$

where $n_{\mathrm{i}}\left(N_{\mathrm{i}}\right.$, respectively) represents the number of colonies (the desired number of seeded cells, respectively) of the flask $i$. We also proposed an estimator to gather the results of independent experiments:

$$
S=\sum_{i=1}^{q} \alpha_{i} S_{i}
$$


with:

$$
\begin{aligned}
& \alpha_{i}=\sigma^{-2}\left(S_{i}\right) / \sum_{i=1}^{q} \sigma^{-2}\left(S_{i}\right) \\
& \left(\frac{\sigma(S)}{\langle S\rangle}\right)^{2}=\left(\frac{\sigma(P)}{\{P\}}\right)^{2}+\left(\frac{\sigma(P E)}{\{P E\}}\right)^{2} \\
& \frac{\sigma(P)}{P}=\frac{1}{\sqrt{\sum_{i=1}^{q} n_{i}}} \text { and } \frac{\sigma(P E)}{P E}=\frac{1}{\sqrt{\sum_{i=1}^{q} n(P E)_{i}}}
\end{aligned}
$$

Here $n(P E)_{\mathrm{i}}$ is defined as the number of colonies counted in the flask " $\mathrm{i}$ " in the experiment for determining $P E$. The standard error for the average survival is:

$$
\sigma(S)=1 / \sqrt{\sum_{i=1}^{q} \sigma^{-2}\left(S_{i}\right)}
$$

In the following results, the error bars refer to $95 \%$ confidence intervals. All these statistical developments were applied to define the experimental conditions that ensured a statistical error of about $\pm 5 \%$, for the determination of survival to photons.

\section{RESULTS}

\section{Determination of the LEM input $\alpha$ and $\beta$ parameters}

The $\alpha$ and $\beta$ input parameters of the LEM were determined from the measurement of survival to photons for both SCC61 and SQ20B cell lines. Colony formation assays used the two protocols described in the Materials and Methods. Each value was averaged over 4-10 independent samples and experiments were performed three times. Error bars were calculated as described above. Figures $1 \mathrm{~A}$ and $1 \mathrm{~B}$ show the dose-response curves for the cell-killing effects of photons on SCC61 and SQ20B cells. As mentioned above, accuracy is essential for any LEM application. Despite the explicit PE corrections in Eq. (5) (Table 1), the PE can suffer from statistical fluctuations. Therefore, we set the survival value at zero dose to be a free parameter. For practical purposes, we added a free constant "c" to the second-order polynomial $\alpha . D+\beta \cdot D^{2}$. We observed low values of $\mathrm{c}$, which demonstrate a good estimation 
of the PE (the corrections to PE were less than 10\%). After renormalization, each dose point was averaged over the different experiments, according to the formulation proposed in the "Statistical Analysis" section. This procedure avoids the usual attribution of the same weight to experimental points despite possible higher error bars. Mathematically speaking, our estimator is more efficient because the standard error is lower. We fitted the LQ model to experimental data using a standard process of minimization (least mean squares). To estimate the reliability of the estimated $\alpha$ and $\beta$ parameters, we shifted some points within their error bars and estimated new values. We observed that the $\beta$ value could vary dramatically, although the $\alpha$ parameter and the general feature of the LQ curve did not change significantly. In some cases, the $\beta$ parameter could be multiplied or divided by a factor of 2 or more, which is a severe problem because this parameter plays a core role in LEM predictions. Indeed, the slope $s_{\max }$ of the extrapolated survival curve was almost proportional to $\beta$ (see Eq. 4). Mathematically, minimization methods are adapted when the number of parameters is low compared with the number of experimental points or when the experimental errors are low. This is not the case for cell-based experiments, for which the maximal covered dose cannot overcome 6-10 Gy, and therefore the number of studied doses ranges between 6 and 10 at most. Moreover, despite an identical number of free parameters, the quadratic polynomial $\alpha . D+\beta . D^{2}$ is more flexible compared, for instance, with a straight line. Therefore, we proposed an alternative method. Instead of fitting an LQ model to the experimental survival $S(D)$, we fitted the straight line $\alpha+\beta . D$ on the experimental curve $\frac{-\ln (S(D))}{D}$. This method presented several advantages. First, it was possible to evaluate the relevance of the LQ model by verifying that the experimental points described a straight line to within fluctuation levels. Second, we could perform a manual estimation of the straight-line parameters and then compare these parameters with the numerical minimization. Last, the estimation of the error bars on $\alpha$ and $\beta$ parameters was straightforward. The new results were better, because the worst uncertainty on $\beta$ parameters was $50 \%$ for one experiment with Method 2 in the SQ20B cells. The results, including $\alpha, \beta$, SF2, dose of 50\% (D50) and 10\% survival (D10), are shown in Table 1 for both cell lines. Figure 1 and Table 1 confirm that the SCC61 cells were more radiosensitive than the SQ20B cells (14). A shoulder was clearly observed for the SQ20B radioresistant cells $(\alpha / \beta \approx 2 \mathrm{~Gy}$ ) so we might expect a higher RBE in response to ions. SQ20B cells displayed similar results with both protocols of seeding and irradiation. Instead, SCC61 parameters varied significantly between the two protocols. The difference observed was 
greater for the $\alpha$ parameter (50\% variation) than for $\beta(32 \%)$. These results highlight the importance of establishing survival curves with a common protocol for photons and ion irradiation in SCC61 cells.

\section{Cell nuclear radius}

The mean radius was $7.1 \pm 0.8 \mu \mathrm{m}$ for the SCC61 cell line and $7.9 \pm 1.1 \mu \mathrm{m}$ for SQ20B.

\section{Radiobiological parameters for the response to ions}

Theoretically, $\operatorname{Ar}[85 \mathrm{MeV} / \mathrm{n}]$ provides a way to increase the LET without changing ion velocity with regard to $\mathrm{C}[72 \mathrm{MeV} / \mathrm{n}]$. On the contrary, comparison of $\operatorname{Ar}[85 \mathrm{MeV} / \mathrm{n}]$ to $\mathrm{C}[9.8$ $\mathrm{MeV} / \mathrm{n}]$ underlines the influence of velocity for two equivalent high-LET ions. This set of ions provides a good test for models. Figures 1C and 1D show the experimental doseresponse curves for these three ions for both cell lines and Table 2 summarizes the radiobiological parameters. As expected, clonogenic survival to $C[72 \mathrm{MeV} / \mathrm{n}]$ was lower than for X-ray exposure. The $\alpha$ value was estimated to be $1.08 \mathrm{~Gy}^{-1}$ for the SCC61 cell line and $0.76 \mathrm{~Gy}^{-1}$ for SQ20B cells. A shoulder could no longer be observed even for SQ20B cells. The relative biological efficiency (RBE) at 10\% survival was 1.5 for the SCC61 cells and 2.1 for SQ20B. In response to $\operatorname{Ar}[85 \mathrm{MeV} / \mathrm{n}]$, the $\alpha$ value was estimated to $1.2 \mathrm{~Gy}^{-1}$ for the SCC61 cell line and $0.85 \mathrm{~Gy}^{-1}$ for SQ20B cells. The RBE was 1.6 for SCC61 cells and 2.4 for SQ20B. The efficiency of $\mathrm{C}[9.8 \mathrm{MeV} / \mathrm{n}]$ with regard to $\mathrm{C}[72 \mathrm{MeV} / \mathrm{n}]$ and $\operatorname{Ar}[85 \mathrm{MeV} / \mathrm{n}]$ was significantly higher. The $\alpha$ value was $2.1 \mathrm{~Gy}^{-1}$ for SCC61 cells and $1.0 \mathrm{~Gy}^{-1}$ for SQ20B, and the RBE values were 4.2 and 2.8, respectively. Whatever the beam used, the SQ20B cell line was more resistant than the SCC61 cells.

\section{Experimental data versus predicted data of LEM}

We next applied the LEM to predict ion-induced cell killing from measurements of the dose-response curves for photons and from the nuclear radius. Because LEM requires a cellline response to X-rays for high doses ( $>10 \mathrm{~Gy}$ ), we extrapolated the photon response to high doses according to the original author's procedure (11) (see Eq. (4)). For both cell lines we chose $\mathrm{D}_{0}=10 \mathrm{~Gy}$, which corresponds to the value reported for the V79 reference cell line (18, 19). The results are presented in Figures $2 \mathrm{~A}$ and $2 \mathrm{~B}$. The LEM prediction did not match the experimental data, although there was a general tendency for agreement with it. To improve the accuracy of prediction, we then allowed $\mathrm{D}_{0}$ to be a free parameter. Table 3 presents the results of $\mathrm{D}_{0}$ optimized for each ion in both cell lines. For the SCC61 cell line, the optimized 
$\mathrm{D}_{0}$ values were similar in response to $\mathrm{C}[72 \mathrm{MeV} / \mathrm{n}]$ and $\operatorname{Ar}[85 \mathrm{MeV} / \mathrm{n}]$, but the values for SQ20B cells differed by $35 \%$. Concerning C $[9.8 \mathrm{MeV} / \mathrm{n}], \mathrm{D}_{0}$ were calculated for the $\alpha$ and $\beta$ parameters of both protocols. The $\frac{D_{0}(\operatorname{method} 2)}{D_{0}(\operatorname{method} 1)}$ ratio was 2.2 for SCC61 cells and 1.4 for SQ20B. Thus, $\mathrm{D}_{0}$ clearly depended on the set of $\alpha$ and $\beta$ parameters used as inputs, even in the SQ20B cell line for which the seeding protocol did not influence cell survival significantly. These results were to be expected, because the survival over the threshold $D_{0}$ is essentially governed by the slope of $s_{\max }=\alpha+2 \beta \cdot D_{0}$. Changing the $\beta$ parameter changed $\mathrm{D}_{0}$ to achieve the same slope. Therefore, we fitted $s_{\max }$ (instead of $\mathrm{D}_{0}$ ), which directly represented the response to photons at high doses. As can be seen in Table 3, the variation of the fitted $s_{\max }$ over all experimental conditions was lower than that for $\mathrm{D}_{0}$. The worst variation was 1.3 in SCC61 cells and 1.1 in SQ20B when comparing Methods 1 and 2 for C[9.8 MeV/n]. Thus, $s_{\max }$ gave a better parameter than $\mathrm{D}_{0}$.

We could now achieve the initial goal of finding parameters for head and neck squamous carcinoma cells applicable to treatment planning in hadrontherapy. We therefore optimized $s_{\max }$ for both cell lines and found that a $2.5 \mathrm{~Gy}^{-1}$ value appeared adequate. The LEM predictions are represented in Figures $2 \mathrm{C}$ and 2D. The agreement between LEM and experimental data was much better than the previous results for $\mathrm{D}_{0}=10 \mathrm{~Gy}$ shown in Figures $2 \mathrm{~A}$ and $2 \mathrm{~B}$. The main trends were respected and the order of magnitude of survival values was more acceptable. Nevertheless, despite this optimization, the LEM model could not predict the cell responses to fast and slow ions with the same accuracy. Although the agreement with $\mathrm{C}[72 \mathrm{MeV} / \mathrm{n}]$ and $\operatorname{Ar}[85 \mathrm{MeV} / \mathrm{n}]$ was reasonable in both cell lines, predictions for $\mathrm{C}[9.8 \mathrm{MeV} / \mathrm{n}]$ overestimated cell killing for the radioresistant SQ20B cell line and underestimated it for the radiosensitive SCC61 cells. Furthermore, increasing $s_{\max }$ to higher levels improved the predictions for $\mathrm{C}[9.8 \mathrm{MeV} / \mathrm{n}]$ specifically in SCC61 cells, but made all the other predictions worse. By contrast, decreasing $s_{\max }$ improved the predictions for C $[9.8$ $\mathrm{MeV} / \mathrm{n}]$ in SQ20B cells, but worsened all the other predictions. Finally, we confirmed that LEM could not predict with the same accuracy the response to fast and slow ions and thus has to be improved (20). This has to be taken into account in treatment planning because most tumors are irradiated by a field of mixed carbon energies. 


\section{DISCUSSION}

Head and neck cancers are often resistant to conventional radiotherapy and are now considered as elective indications for hadrontherapy with carbon ions (21). The present study had two specific aims. - first, to establish the radiobiological parameters of HNSCC in response to ions of three different values of LET and velocity; - second, to evaluate the relevance of the LEM in these cells. We chose carbon ions with two extreme velocities. $\operatorname{Ar}[85$ $\mathrm{MeV} / \mathrm{n}]$ provides one way to increase the LET without changing ion velocity with regard to $\mathrm{C}[72 \mathrm{MeV} / \mathrm{n}]$. Moreover, a comparison of Ar with $\mathrm{C}[9.8 \mathrm{MeV} / \mathrm{n}]$ displays the influence of velocity for two equivalent LET values. Therefore, this set of ions provides a good test for such models. Irradiations were performed in track-segment conditions to allow direct comparisons of theory and experimental data. Cell survival was chosen as a classical biological endpoint (22), which up to now is the most appropriate to estimate tumor control probability. The results bring a high confidence for the effective treatment of head and neck tumors resistant to photons by carbon hadrontherapy because the RBE at $10 \%$ of survival reached 4 and 3 in SCC61 and SQ20B cells, respectively, for C[9.8 MeV/n], which corresponds to energy at the Bragg peak. One could thus expect a favorable ratio between tumor control and healthy tissue complications. We also confirm that the biological effects of ions depend not only on the dose, but also on ion velocity and nature (4). High LET radiations do not eliminate the radiobiological properties observed at low LET values because the SCC61 cell line was still more radiosensitive than the SQ20B cell line in response to carbon irradiation. This might indicate that the mechanisms of cell death may not differ strongly between low and high LET radiations.

To date, two approaches have been developed for planning hadrontherapy treatment: the LEM adapted to active beam control at GSI (8) and the procedure of HIMAC (Heavy Ion Medical Accelerator in Chiba, Japan) adapted to a passive beam (23). The latest procedure is based on similarities in the biological response to carbon and neutron beams of the same LET. It consists in interpolating the $\alpha$ and $\beta$ coefficients of the LQ model $(23,24)$. In the clinical approach used at GSI, the "microscopic" effect of ion tracks is assumed equal to that of corresponding photon doses (6). The key point of LEM is to predict cell survival to any ion irradiation on the basis of three independent datasets: (i) the cell survival response to photon irradiation; (ii) the cell nuclear radius, which is supposed to be the main sensitive target of the cell; and (iii) the radial dose distribution inside the particle track, which depends on ion atomic number and energy (8). Dose-response studies with carbon ions have been performed 
in several human and mammalian cell lines $(9,25,26)$; however, no previous paper has applied the LEM to experimental data in human tumor cells within track segment conditions.

We have evaluated cell survival in response to X-rays, and applied LQ model to estimate $\alpha$ and $\beta$ parameters. Following LEM's authors, we extrapolated dose-response curve beyond the dose $D_{0}$ (Eq. 4). We observed that, for a fixed value of $D_{0}$, the prediction of cell survival to ions depended significantly on the $\beta$ parameter. Multiplying $\beta$ by a factor of 1.5 modified the slope of the survival curve for $\mathrm{C}[9.8 \mathrm{MeV} / \mathrm{n}]$ and $\mathrm{C}[72 \mathrm{MeV} / \mathrm{n}]$ by $50 \%$. Therefore, we developed a method and statistical estimators to decrease the uncertainty for $\beta$ parameters. The linear extrapolation plays a major role and cannot be arbitrarily set. We fitted the value of $\mathrm{D}_{0}$, which sets the parameters for the linear extrapolation, to obtain an optimized agreement between the experimental results and LEM predictions. This procedure was probably applied by the original LEM authors (6), because they proposed a different threshold dose value for each cell line. We further showed that the slope of the linear extrapolation was a much better parameter than $\mathrm{D}_{0}$. By definition (see Eq. 4), the number of lethal events at high local doses is mainly determined by this slope. Moreover, with this parameterization, the predictions were less sensitive to $\beta$. For instance, multiplying $\beta$ by a factor of 2 modified the slope of the survival curve for $\mathrm{C}[9.8 \mathrm{MeV} / \mathrm{n}]$ and $\mathrm{C}[72 \mathrm{MeV} / \mathrm{n}]$ by less than $10 \%$. Reducing the effect of $\beta$ increased the reliability of the parameterization, because we have shown the difficulty in determining the $\beta$ parameter experimentally. For both SQ20B and SCC61 cell lines, the optimized slope was found to be $2.5 \mathrm{~Gy}^{-1}$. The value of the slope $s_{\max }$ for SQ20B and SCC61 cells differed from adenoid cystic carcinoma cells (27), another type of head and neck tumor, but is consistent with that for chordoma cells. These similarities and differences mimic the features of the response to photons radiations. Indeed, the $\alpha$ and $\beta$ parameters for adenoid cystic carcinoma cells in response to photon radiations differed strongly from those of squamous carcinoma SQ20B and SCC61 cells whereas they were very similar to that of chordoma cells. This might indicate that establishing a survival curve in response to X-rays could help in fixing the slope $s_{\max }$.

Using a slope of $2.5 \mathrm{~Gy}^{-1}$, LEM could predict the main trends of experimental measurements. However, the killing efficiency of $\mathrm{C}[9.8 \mathrm{MeV} / \mathrm{n}]$ was underestimated for the radiosensitive cell line and overestimated for the radioresistant cell line. This inaccuracy could have been because a different protocol was applied for the $\mathrm{C}[9.8 \mathrm{MeV} / \mathrm{n}]$ beam, but we performed the LEM calculations using as input parameters the $\alpha$ and $\beta$ values extracted from X-ray survival curves obtained with the same protocol. Second, we found that the protocol did 
not play any significant role, at least for the SQ20B cell line. The difficulty to get simultaneously an accurate agreement of the theory with experimental data for both the highLET and low-LET ions has been reported for proton beams in V79 Chinese hamster ovary (CHO) cells (19) and for carbon and oxygen beams in CHO cells (11). Finally, our measurements strengthen the need for further studies on the LEM to improve the agreement between experimental data and theory over a large set of projectiles. On this aspect, Elsässer and Scholz (28) have proposed a correction to the linear extrapolation of the survival to Xrays at very high dose to take into account some effects of cluster damage. This modification introduced as a new parameter the maximal distance for which two or more single-strand DNA breaks give one double strand break. After modifying the shape of the local dose in the ion track and fitting a new value for $\mathrm{D}_{0}$, they obtained predictions that better matched with the experimental data for both low- and high-LET.

From this analysis, we conclude that it is not possible simply to take advantage of the $\alpha$ and $\beta$ parameters established for X-ray treatment to deduce appropriate planning for treatment with carbon ions, considering the requirement for an optimized value of $s_{\max }$ or $\mathrm{D}_{0}$. Ion data from various tumor cell types are required to optimize individual treatment by carbon hadrontherapy. 


\section{FIGURE LEGENDS}

Figure 1: Dose-response curves for killing of SCC61 (left panels) and SQ20B cells (right panels) to X-ray (A, B) and ions (C, D): X-ray with method1 (full squares); X-ray with method 2 (open squares); $\mathrm{C}[72 \mathrm{MeV} / \mathrm{n}]$ (open circles); $\operatorname{Ar}[85 \mathrm{MeV} / \mathrm{n}]$ (full triangles); $\mathrm{C}[9.8 \mathrm{MeV} / \mathrm{n}]$ (open diamonds).

Figure 2: Comparisons of LEM predictions for the experimental data of Figures 1C and 1D for SCC61 (left panels) and SQ20B cells (right panels) Calculations are performed using $\mathrm{D}_{0}=$ $10 \mathrm{~Gy}(\mathrm{~A}, \mathrm{~B})$ and $s_{\max }=2.5 \mathrm{~Gy}^{-1}$ (C, D). Experiments: C[72 MeV/n] (open circles); $\operatorname{Ar}[85$ $\mathrm{MeV} / \mathrm{n}]$ (full triangles); $\mathrm{C}[9.8 \mathrm{MeV} / \mathrm{n}]$ (open triangles). For $\mathrm{C}[9.8 \mathrm{MeV} / \mathrm{n}]$, LEM was applied to the results of photons curves obtained with the two methods of cell seeding (Methods 1 and 2). 


\section{REFERENCES}

1. Debus J, Jackel O, Kraft G, et al. Is there a role for heavy ion beam therapy? Recent Results Cancer Res 1998;150:170-182.

2. Brahme A, Nilsson J, Belkic D. Biologically optimized radiation therapy. Acta Oncol 2001;40:725-734.

3. Krengli M, Orecchia R. Medical aspects of the National Centre For Oncological Hadrontherapy (CNAO-Centro Nazionale Adroterapia Oncologica) in Italy. Radiother Oncol 2004;73:21-23.

4. Kraft G. Tumor therapy with heavy charged particles. Prog Part Nucl Phys 2000;45:473544.

5. Crespo P, Shakirin G, Enghardt W. On the detector arrangement for in-beam PET for hadron therapy monitoring. Phys Med Biol 2006;51:2143-2163.

6. Kramer M, Scholz M. Treatment planning for heavy-ion radiotherapy: calculation and optimization of biologically effective dose. Phys Med Biol 2000;45:3319-3330.

7. Kramer M, Scholz M. Rapid calculation of biological effects in ion radiotherapy. Phys Med Biol 2006;51:1959-1970.

8. Goodhead DT. Mechanisms for the biological effectiveness of high-LET radiations. $J$ Radiat Res (Tokyo). 1999;40:1-13.

9. Scholz M, Kraft G. Track structure and the calculation of biological effects of heavy charged particles. Adv Space Res 1996;18:5-14.

10. Katz R, Zachariah R, Cucinotta FA, et al. Survey of cellular radiosensitivity parameters. Radiat Res;1994;140:356-365. 
11. Scholz M, Kellerer AM, Kraft-Weyrather W, et al. Computation of cell survival in heavy ion beams for therapy. The model and its approximation. Radiat Environ Biophys 1997;36:59-66.

12. Ziegler JF. The Stopping and Range of Ions in Matter. Vol. 1, New York, Pergamon Press, 1985.

13. Weichselbaum RR, Clark JR, Miller D, et al. Combined modality treatment of head-andneck cancer with cisplatin, bleomycin, methotrexate-leucovorin chemotherapy. Cancer $1985 ; 55: 2149-2155$.

14. Alphonse G, Aloy MT, Broquet $\mathrm{P}$, et al. Ceramide induces activation of the mitochondrial/caspases pathway in Jurkat and SCC61 cells sensitive to gamma-radiation but activation of this sequence is defective in radioresistant SQ20B cells. Int J Radiat Biol 2002;78:821-835.

15. Henshaw DL. Applications of CR-39 nuclear track detector in medicine and technology. Phys Tec 1982;13:266-227.

16. Kraft G, Daues H, Fischer B et al. Irradiation chamber and sample changer for biological samples Nuc Inst and Meth 1980;168:175-179.

17. Puck TT, Marcus PI. A rapid method for viable cell titration and clone production with Hela cells in tissue culture: the use of $\mathrm{x}$-irradiated cells to supply conditioning factors. Proc Natl Acad Sci U S A 1955;41:432-437.

18. Kraft G., Scholz M. On the parameterization of the biological effect in a mixed radiation field Adv Space Res 1994;14:997-1004.

19. Paganetti H, Goitein M. Biophysical modelling of proton radiation effects based on amorphous track models. Int J Radiat Biol 2001;77:911-928.

20. Elsasser T, Scholz M. Improvement of the local effect model (LEM)-implications of clustered DNA damage. Radiat Prot Dosimetry. 2006;122:475-477. 
21. Mizoe JE, Tsujii H, Kamada T, et al. Dose escalation study of carbon ion radiotherapy for locally advanced head-and-neck cancer. Int J Radiat Oncol Biol Phys 2004;1:60:358-364.

22. Webb S, Nahum AE. A model for calculating tumour control probability in radiotherapy including the effects of inhomogeneous distributions of dose and clonogenic cell density. Phys Med Biol 1993;38:653-666.

23. Kanai T, Furusawa Y, Fukutsu K, et al. Irradiation of mixed beam and design of spreadout Bragg peak for heavy-ion radiotherapy. Radiat Res 1997;147:78-85.

24. Kanai T, Endo M, Minohara S, et al. Biophysical characteristics of HIMAC clinical irradiation system for heavy-ion radiation therapy. Int $J$ Radiat Oncol Biol Phys 1999;44:201-210.

25. Tsuruoka C, Suzuki M, Kanai T, et al. LET and ion species dependence for cell killing in normal human skin fibroblasts. Radiat Res 2005;163:494-500.

26. Weyrather WK, Ritter S, Scholz M, et al. RBE for carbon track-segment irradiation in cell lines of differing repair capacity. Int J Radiat Biol 1999;75:1357-1364.

27. Battermann JJ, Breur K, Hart GA and al. Observations on pulmonary metastases in patients after single doses and multiple fractions of fast neutrons and Co-60 gamma rays. Eur J Cancer 1981;17 539-548.

28. Elsasser T, Scholz M. Cluster effects within the local effect model. Radiat Res 2007;167:319-329. 
Table 1: Parameters of the linear-quadratic model obtained for SCC61 and SQ20B cell lines irradiated with photons by methods 1 and 2 .

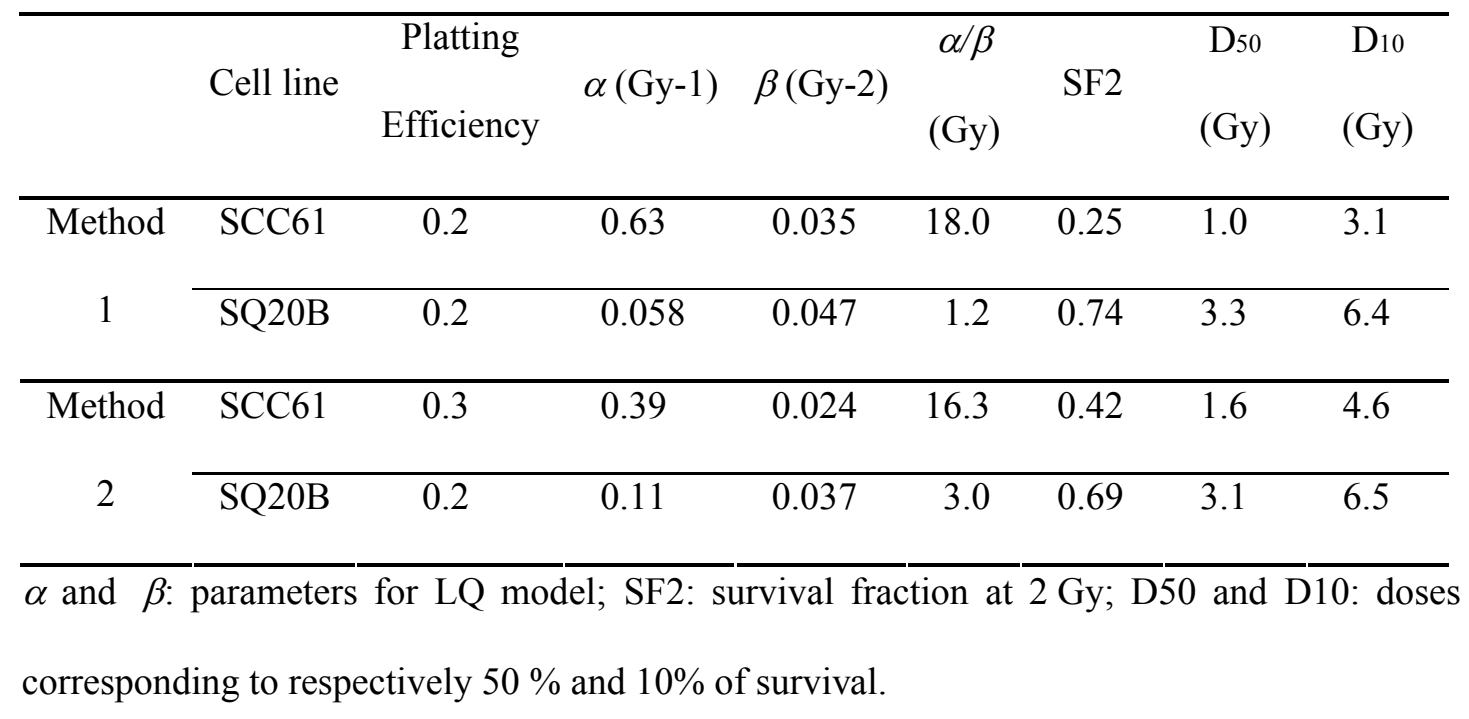


Table 2: Biological parameters of SCC61 and SQ20B cell lines irradiated with Carbon[72 $\mathrm{MeV} / \mathrm{n}]$, Argon[85 MeV/n] and Carbon[9.8 MeV/n].

\begin{tabular}{|c|c|c|c|c|c|c|}
\hline Radiation & Cell line & SF2 & $\alpha(\mathrm{Gy}-1)$ & D50 (Gy) & D10 (Gy) & RBE \\
\hline \multirow{2}{*}{ Carbon $72 \mathrm{MeV} / \mathrm{n}$} & SCC61 & 0.11 & 1.08 & 0.64 & 2.1 & 1.5 \\
\hline & SQ20B & 0.23 & 0.76 & 0.91 & 3.0 & 2.1 \\
\hline \multirow{2}{*}{ Argon $85 \mathrm{MeV} / \mathrm{n}$} & SCC61 & 0.09 & 1.2 & 0.58 & 1.9 & 1.6 \\
\hline & SQ20B & 0.18 & 0.85 & 0.82 & 2.7 & 2.4 \\
\hline \multirow{2}{*}{ Carbon $9.8 \mathrm{MeV} / \mathrm{n}$} & SCC61 & 0.015 & 2.1 & 0.33 & 1.1 & 4.2 \\
\hline & SQ20B & 0.13 & 1.0 & 0.69 & 2.3 & 2.8 \\
\hline
\end{tabular}


Table 3: Values of the $D_{0}$ parameter obtained by fitting LEM results to experimental data for ion irradiation of SCC61 and SQ20B cell lines. For carbon [9.8 MeV/n], $D_{0}$ is given for the set of LQ parameters of Methods 1 and 2.

\begin{tabular}{ccccc}
\hline & \multicolumn{3}{c}{ SCC61 } & \multicolumn{2}{c}{ SQ20B } \\
\hline Radiation & $\mathrm{D}_{0}$ & $s_{\max }$ & $\mathrm{D}_{0}$ & $s_{\max }$ \\
\hline $\mathrm{C}[72 \mathrm{MeV} / \mathrm{n}](1)$ & 25 & 2,4 & 37 & 3,5 \\
\hline $\mathrm{Ar}[85 \mathrm{MeV} / \mathrm{n}](1)$ & 28 & 2,6 & 26 & 2,5 \\
\hline $\mathrm{C}[9.8 \mathrm{MeV} / \mathrm{n}](1)$ & 38 & 3,3 & 19 & 1,8 \\
\hline $\mathrm{C}[9.8 \mathrm{MeV} / \mathrm{n}](2)$ & 82 & 4,3 & 26 & 2,0 \\
\hline
\end{tabular}


Figure 1

Scc61

A Dose (Gy)

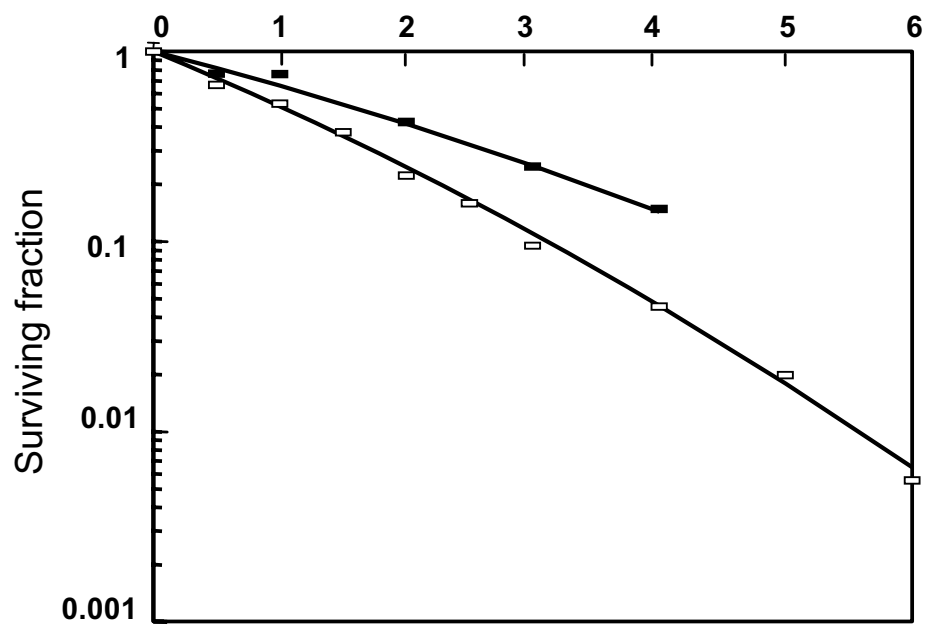

C

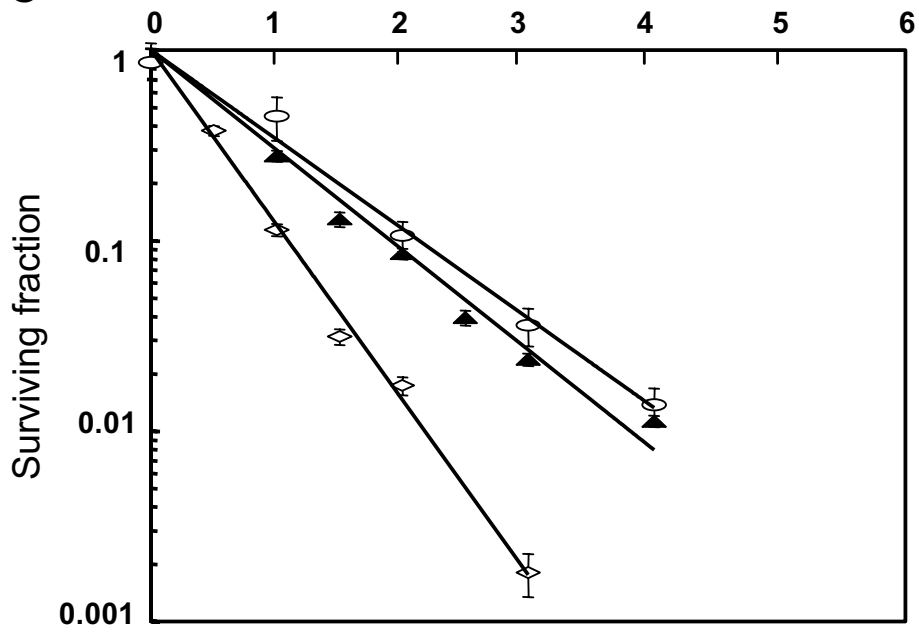

SQ20B

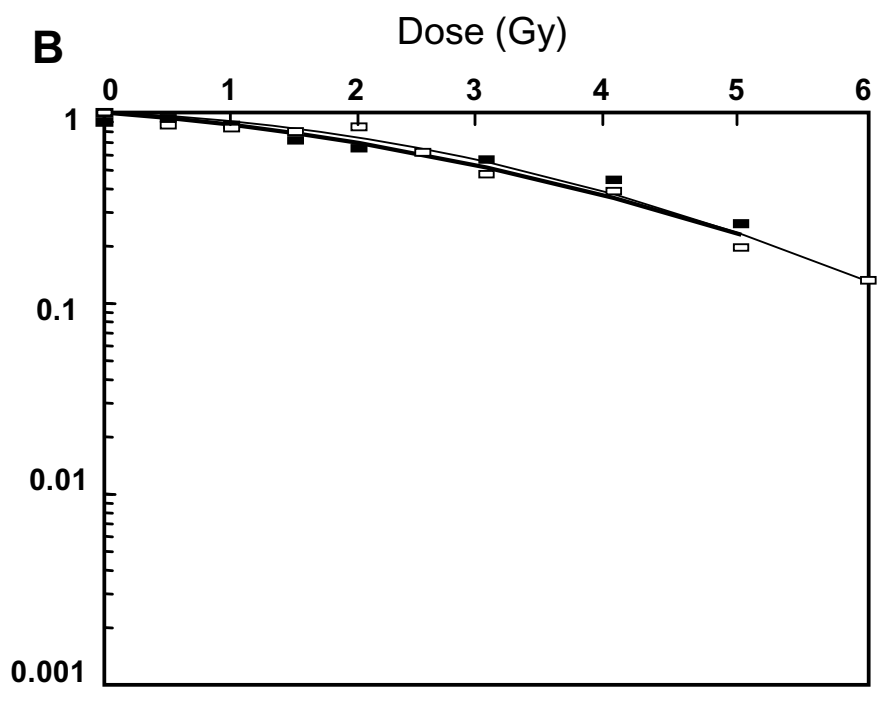

D

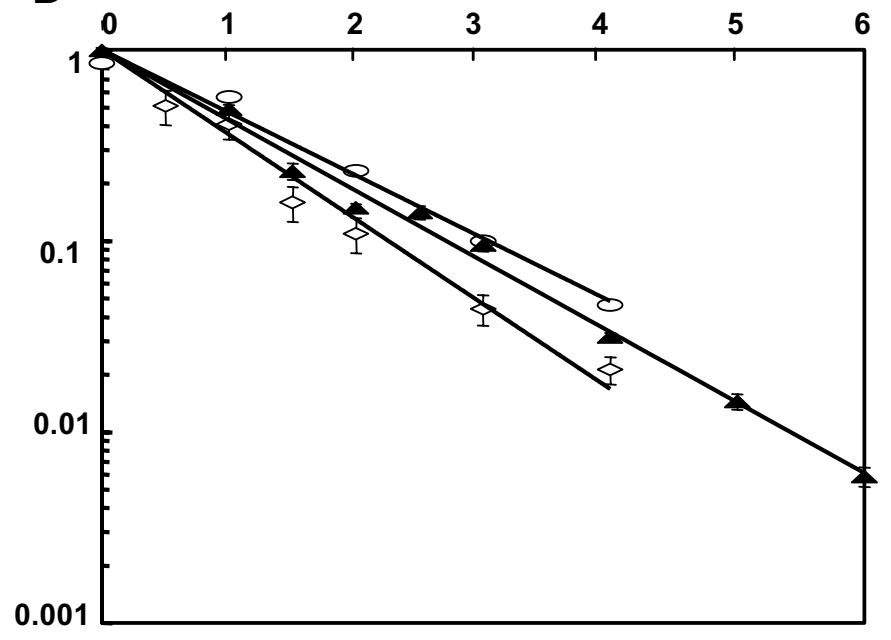


A

Dose (Gy)

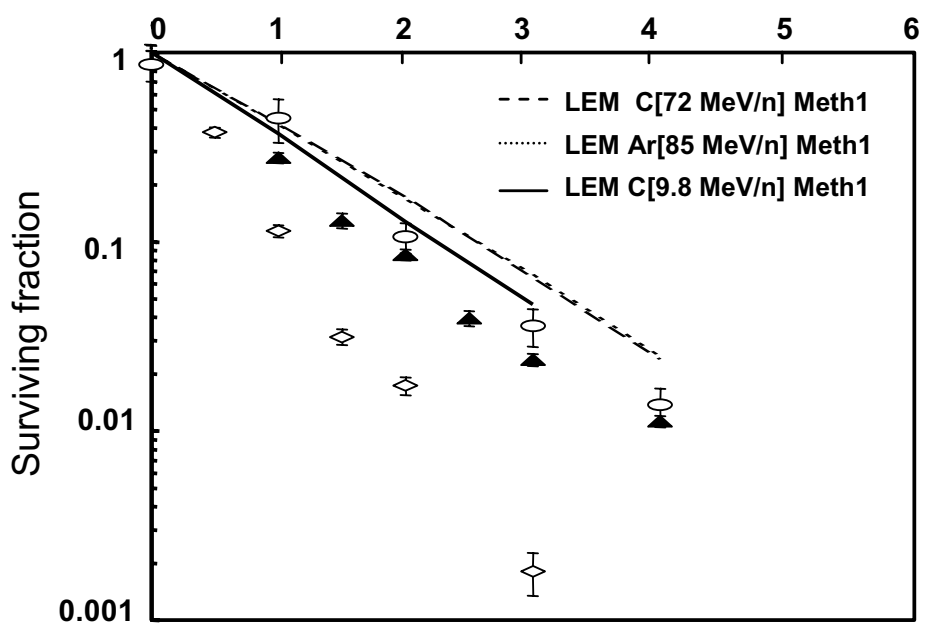

C

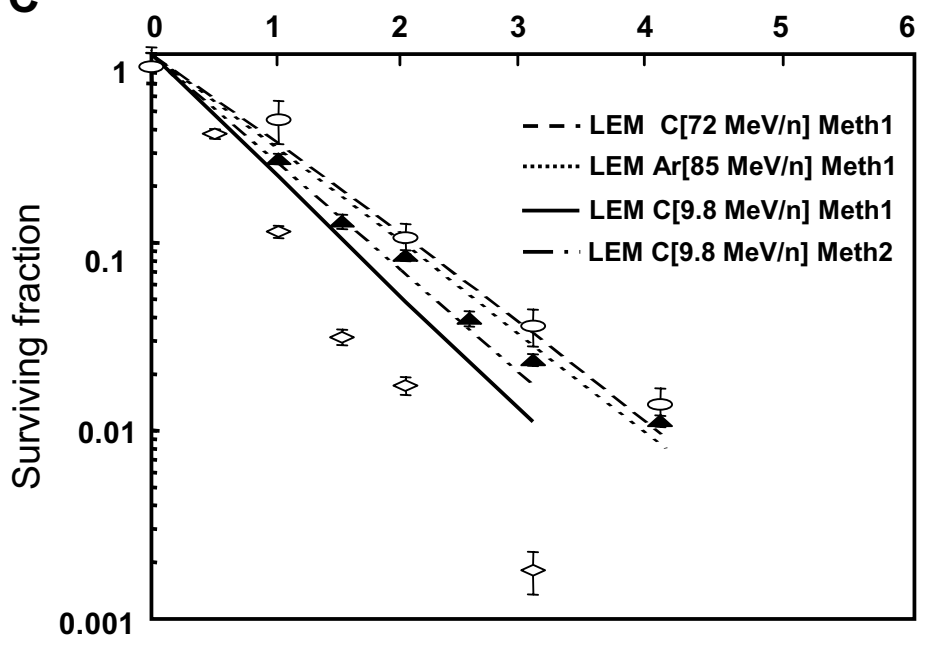

B

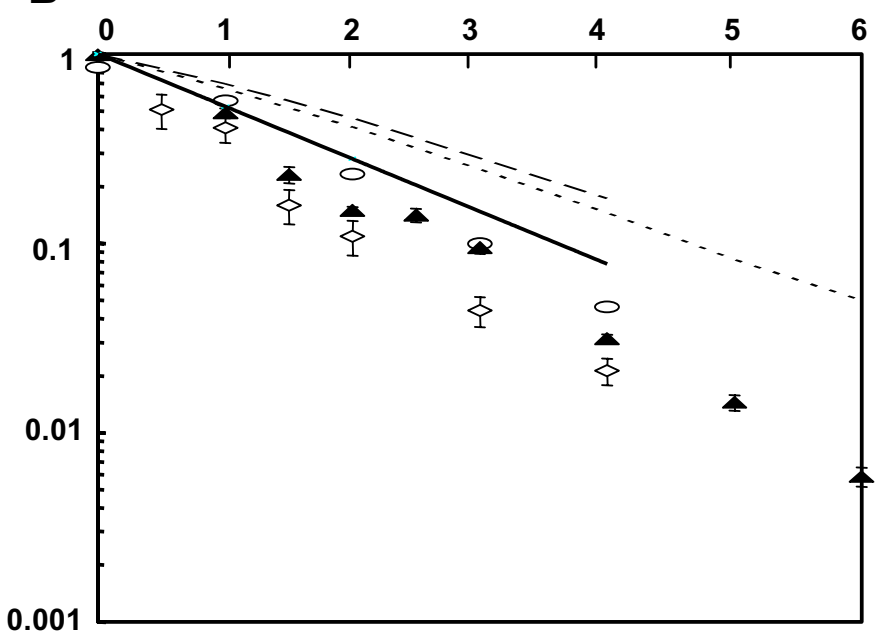

D

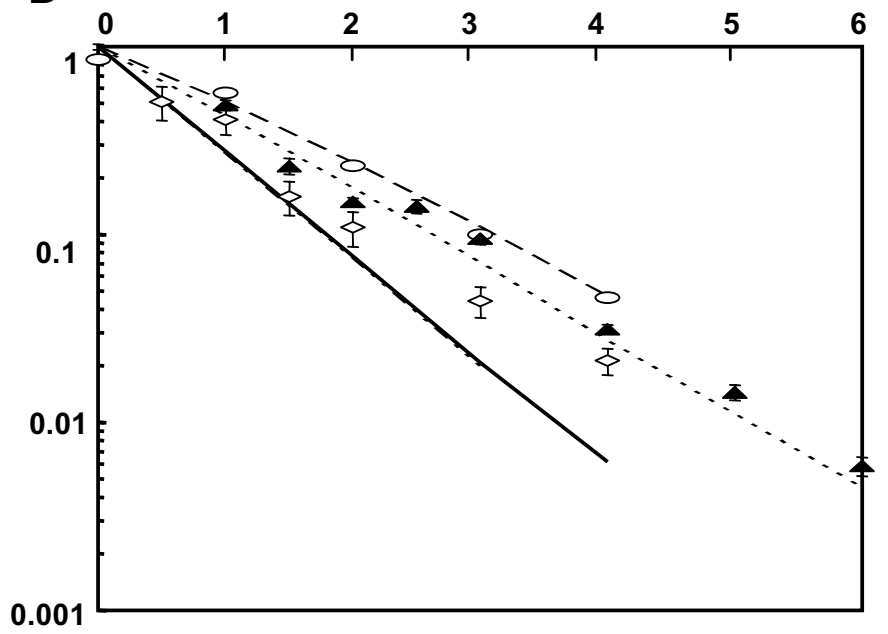

